\title{
Lipid-Based Nano Formulation Approach to Target Brain for the Management of Tuberculosis through Intranasal Delivery: Formulation Development and Evaluation
}

\author{
Sai Surya Nikitha Upadhyayula, Raman Sureshkumar*, S K Janani, Chenmala Karthika \\ Department of Pharmaceutics, JSS College of Pharmacy Ooty, JSS Academy of Higher Education and Research, \\ Ooty, Nilgiris, Tamil Nadu, INDIA.
}

\begin{abstract}
Objectives: Development of nano-emulsion based drug delivery as combined drug loaded form for the possible management of meningitis. Intranasal delivery of nano-emulsion to the brain and study the nasal irritancy. Methodology: Spontaneous emulsification method was used in preparing the nanoemulsion. The compatibility studies i.e., Differential Scanning Colorimetry and Fourier Transform Infrared Spectroscopy were carried out for Isoniazid, Rifampicin, Resveratrol and physical mixture. Pseudoternary phase diagrams were constructed in various ratios. The formulations which passed the thermodynamic stability studies were evaluated for particle size, zeta potential and poly dispersity index. Cytotoxicity of the pure drugs and formulations were measured in in vitro cell line. The formulations were given for histopathological studies. Then these formulations were administered to rats intranasally and the brain of the rats was collected. Then the brain tissue was minced and was given for LCMS analysis to know the amount of drug reaching the brain. Results: The DSC and FTIR graphs state that the drugs were compatible with each other. The formulations which passed the thermodynamic stability studies were A7, A9, J8 and G9. Among these formulations A7 was selected as it had lower particle size when compared to other formulations. The formulations showed low nasal irritancy and no malignancy. From the LCMS analysis it was found that $82.6 \%$ of Isoniazid with Resveratrol nanoemulsion and $78.48 \%$ of Rifampicin with Resveratrol nanoemulsion has reached the targeted area the brain. Conclusion: From the studies it can be concluded that when the formulations were administered intranasally maximum amount of the drugs had reached the targeted area the brain. So, when the drugs are given intranasally there could be decrease in the dose of the drug, increased bioavailability at the site of action and increased therapeutic efficacy.
\end{abstract}

Key words: Tuberculous meningitis, Intranasal delivery, Nanoemulsion, Blood Brain Barrier, Mycobacterium tuberculosis.

\section{INTRODUCTION}

Tuberculosis is caused by the bacteria mycobacterium tuberculosis. ${ }^{1}$ It may affect any part of the body but it primarily affects the lungs which is termed as pulmonary tuberculosis. ${ }^{2}$ It usually enters the oxygenated regions of the body. As the brain is the most oxygenated region of the body the bacteria also enter the brain causing tuberculous meningitis. The main area of the brain which gets affected is the sub-arachnoid space. There are many drugs available for the treatment of tuberculosis. The prominently used drugs are the first line drugs isoniazid, rifampicin, pyrazinamide and ethambutol. ${ }^{3,4}$ In the case of TBM pyrazinamide is replaced with streptomycin as the former cannot cross the BBB. ${ }^{5}$ In some cases, the patients are resistant to the first-
Submission Date: 25-09-2019; Revision Date: 26-12-2019; Accepted Date: 26-02-2020

DOI: 10.5530/ijper.54.2s.75 Correspondence: Dr. Raman Sureshkumar, Department of Pharmaceutics, JSS College of Pharmacy Ootacamund, JSS Academy of Higher Education and Research, Mysuru-570015, Karnataka, INDIA.

Phone: $+91-9865064872$ E-mail: sureshcoonoor@ jssuni.edu.in

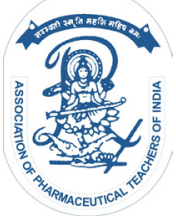

www.ijper.org 
line drugs especially isoniazid and rifampicin. This is termed as Multi-drug Resistant Tuberculosis. In this case the patients are treated with second line drugs which exhibit more side effects when compared to the firstline drugs. in some cases, the patients are resistant to both first-line drugs and fluoroquinolones. ${ }^{6}$ This condition is termed as extensively drug resistant tuberculosis. In this case the hospitalisation of the patient is required and the drugs are administered parenterally. The main reason for the occurrence of MDR Tb and XDR Tb are irregular intake of the drugs by the patients. ${ }^{1,7}$ The main hurdle in the treatment of TBM is the presence of the blood brain barrier. The BBB doesn't allow the foreign substances to enter the brain. So, due to this reason many drugs cannot cross the $\mathrm{BBB}$ and reach the brain. ${ }^{8,9}$ Even the presence of the blood-cerebrospinal fluid barrier also restricts the entry of substances into the brain. The complications occurring in patients may be due to the medications such as hepatotoxicity, peripheral neuropathy, rashes and retinopathy or they may be long-term complications such as cognitive impairment, epilepsy, severe hearing loss, hypothalamic dysfunction and dysphasia. ${ }^{10,11}$

The main problem associated with the current drugs is that the maximum amount of drug cannot cross the Blood Brain Barrier and reach the targeted site the brain. It is because the blood brain barrier is a tight junction and it won't allow the substances to cross it. The high dose of the drugs and the long duration of treatment is also another problem which causes many side effects. ${ }^{7,9}$ So, due to this reason nanotechnology-based approach has emerged to decrease the dose of the drugs and its treatment period.

In our study we have selected intranasal delivery of the formulation as there will be direct transport of the drugs from the nasal cavity to the brain. The drugs reach the brain through the olfactory route. This is due to the presence of the olfactory nerves in the brain. Nanoemulsion formulation was used to administer the drugs to the brain. Nano emulsion formulation was selected because of the lower particle size and are pharmacokinetically stable. These formulations can be administered easily through the intranasal route and maximum amount of the drug can reach the targeted area the brain. Flavonoid resveratrol was used in combination with isoniazid and rifampicin individually. It was used as the flavonoids exhibits the antibacterial as well as antiinflammatory activities. Hence, can reduce the dose of the drugs, decrease the time period of the treatment and increase the therapeutic efficacy.

\section{MATERIALS AND METHODS}

\section{Materials}

Isoniazid was obtained from Avra, rifampicin, resveratrol, Kolliphor, Methanol, Di-methyl sulfoxide and Castor oil were obtained from Sigma Ltd, India. Lauroglycol, Transcutol, Labrafil M, Capryol 90 were obtained as gift samples from Gattefosse. Ethanol, Oleic acid, Isopropyl myristate, Tween 80 and potassium chloride were purchased from Avra. All the chemicals obtained were pure and were of laboratory grade.

\section{Methods}

\section{Construction of Standard Calibration Curve}

Calibration curves for the drugs are plotted to know the linearity of the drug when observed at different concentrations by using UV Visible spectrophotometer. The calibration curve was plotted for drugs isoniazid, rifampicin and a flavonoid resveratrol. Then the solutions of the drugs were prepared in $20 \mathrm{mcg} / \mathrm{ml}, 40 \mathrm{mcg} /$ $\mathrm{ml}, 60 \mathrm{mcg} / \mathrm{ml}, 80 \mathrm{mcg} / \mathrm{ml}$ and $100 \mathrm{mcg} / \mathrm{ml}$ concentrations and the absorbance values obtained were plotted in the graphs to check the linearity. The wavelength of isoniazid, rifampicin and resveratrol are $274 \mathrm{~nm}, 374$ and $358 \mathrm{~nm}$ respectively.

\section{Compatibility studies}

\section{Differential Scanning calorimetry}

The DSC was carried for the individual samples of isoniazid, rifampicin, resveratrol as well as for the physical mixture of all the three drugs in the ratio of 1:1:1. It was carried out to know the purity and compatibility of the samples by taking into account he melting point of the drugs and running the samples for a specific temperature range.

\section{Fourier Transform Infrared Spectroscopy}

FTIR studies were carried out to know the functional groups present and to $\mathrm{k}$ now know the compatibility of the drugs. The FTIR studies were carried out for isoniazid, rifampicin and resveratrol and the physical mixture of all the three in the ration 1:1:1.

\section{Screening of oils}

The drugs rifampicin and resveratrol were screened in various oils and the amount of drug soluble in particular oil was noted. The oil in which maximum amount of drug was soluble was selected.

\section{Screening of surfactants and cosurfactants}

Surfactants are used in the formulations to decrease the interfacial tension between the two phases. So, due to this reason many surfactants were screened and the sur- 
factant which was miscible with the oil was selected. Cosurfactants are used in the formulations to decrease the concentration of the surfactants. So, due to this reason many co-surfactants were screened and the one which was miscible with the oil as well as the surfactant was selected.

\section{Construction of pseudoternary phase diagrams}

Pseudoternary phase diagrams were constructed to know the area of the formed nanoemulsion so that the nanoemulsion forming a maximum area can be selected. So for this reason various ratios of the formulations were selected starting from the Smix ratio !:1 to $1: 9$ (!:1, 1:2, 1:3, 1:4, 1:5, 1:6,1:7, 1:8 and 1:9) and 2:1. The diagrams were constructed using CHEMIX software. Hence, the formulation having a maximum area of the nanoemulsion region were selected for the further studies.

\section{Characterization of Nanoemulsion}

\section{Thermodynamic stability study}

Heating and cooling cycle: First the selected formulations were exposed to cold temperature of -4 degree centigrade for $72 \mathrm{hr}$. Then these formulations were kept at 45 degree centigrade for $72 \mathrm{hr}$. The formulations which were stable after the heating and cooling cycle were selected for the further studies.

Centrifugation: The selected formulations from the heating and cooling cycle were centrifuged at $3500 \mathrm{rpm}$ for $10 \mathrm{~min}$. The formulations which were stable were selected for freeze thaw cycle.

Freeze thaw cycle: The stable samples from the centrifugation were exposed to temperatures between $-21^{\circ} \mathrm{C}$ and $+25^{\circ} \mathrm{C}$ for $48 \mathrm{hr}$ respectively. The samples which were stable for these temperatures were selected for the further study. The samples were observed for phase separation or precipitation.

\section{Particle size, zeta potential and poly dispersity index}

The particle size, zeta potential and poly dispersity index of the selected formulations were tested using malvern zeta sizer. The particle size of the formulations was expected to be less than $200 \mathrm{~nm}$, zeta size ranging between -30 to $+30 \mathrm{mV}$ and poly dispersity index which should less than 1 .

\section{Preparation of Nanoemulsion Formulations Preparation of isoniazid- resveratrol nanoemulsion}

The optimised ratio of 1:7 (1:1) was selected for the preparation of nanoemulsion. Depending on the ratio the weighed quantity of resveratrol was dissolved in required amount of oil and isoniazid was dissolved in the optimised amount of water which was to be added to form a nanoemulsion. Surfactant and cosurfactant were added in the specified quantity. To this calculated amount of water was added according to the values from the pseudoternary phase diagrams. Then the formulation was vortexed to form a stable nanoemulsion.

\section{Preparation of rifampicin- resveratrol nanoemulsion}

The same optimised ratio of 1:7(1:1) was selected for the preparation of the nanoemulsion. Then the weighed quantity of rifampicin and resveratrol were dissolved in specified amount of oil depending on its solubility. To this the optimised amount of surfactant and cosurfactant were added. The calculated amount of water to form a nanoemulsion was added from the pseudoternary phase diagrams. Then the formulation was vortexed for proper mixing and to form a stable nanoemulsion.

\section{Cell-line studies for toxicity ${ }^{\mathbf{1 2}}$}

C-6 cell-line of rat glioma was used for finding the toxicity of the drug when targeted to the brain. 5 samples were tested for the toxicity and $\mathrm{IC}_{50}$ values. Rifampicin pure drug, isoniazid pure drug, resveratrol, nanoemulsion of rifampicin and resveratrol and isoniazid and resveratrol were tested. It was done to know the dose of the drug to be administered.

\section{Nasal toxicity Studies ${ }^{13,14}$}

The nasal irritancy studies were carried out using the mucous membrane of the goat. The mucous membrane was collected from the nearby slaughter house. This was stored in phosphate buffer solution till the experiment was carried out. The membrane was washed gently with distilled water. Then the membrane was treated with $2 \mathrm{ml}$ of phosphate buffer solution. The membrane was divided into three parts and one part each was put into placebo nanoemulsion, nanoemulsion of resveratrol and isoniazid and nanoemulsion of resveratrol and rifampicin. These membranes were immersed in the solution for two hours. Then these mucous membranes were stored in 10\% formalin solution and were sent for histopathological studies.

\section{In-vivo studies}

For this study 6 female wistar rats were used. The animals were procured after getting approval from Institutional Animal Ethical Committee. These animals were divided into two groups. The first group was given a combination of isoniazid and resveratrol and the second group was given a combination of rifampicin and resveratrol. These formulations were administered intranasally using a micropipette. 


\section{Homogenisation of the brain}

The rats were euthanised and the brain tissue of the rats was collected and was dissolved in required quantity of $10 \%$ potassium chloride. Then the tissue was homogenised till a white smooth mixture was formed. The volume of the mixture was measured and equal volume of ethanol was added and mixed well. Then the mixture was sonicated and centrifuged. The supernatant obtained after ce, nitrification was collected and subjected to analysis by using LCMS.

\section{Evaluation of brain samples using LC-MS/MS Method development}

The LCMS method was developed using all the stock solutions of the standard drugs isoniazid, rifampicin and flavonoid resveratrol. The mobile phase used in the method development was ammonium acetate buffer.

\section{Evaluation of samples}

The quantitative analysis of the samples was done using the LCMS. After the method development the samples were evaluated using the LCMS/MS by diluting the samples if necessary. Then the graphs of the samples were obtained. The solvent used for this purpose was methanol as mentioned in the Figures 19, 20, 21.

\section{RESULTS}

\section{Standard Calibration Curve}

\section{Calibration curve of Rifampicin}

Linearity for the drug rifampicin was obtained at 10-50 $\mu \mathrm{g} / \mathrm{ml}$ and this linearity was used for determining the concentration as denoted in Figure 1.

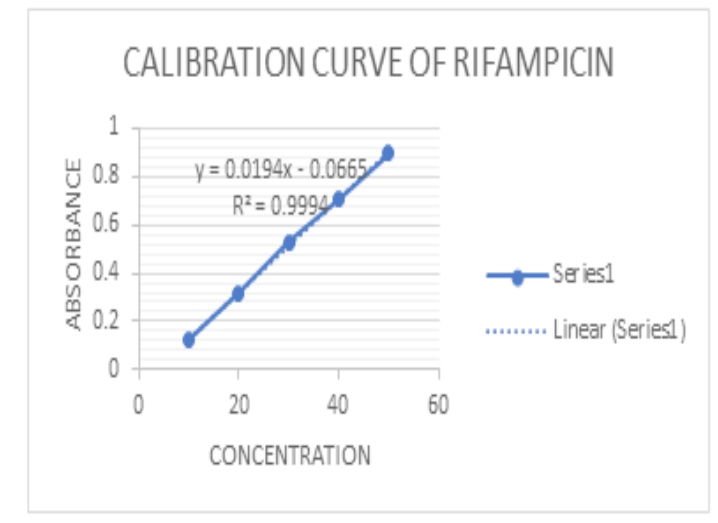

Figure 1: Calibration curve of Rifampicin.

\section{Calibration curve of Isoniazid}

Linearity for the drug isoniazid was obtained at 10-50 $\mu \mathrm{g} / \mathrm{ml}$ and this linearity was used for determining the concentration as represented in Figure 2.

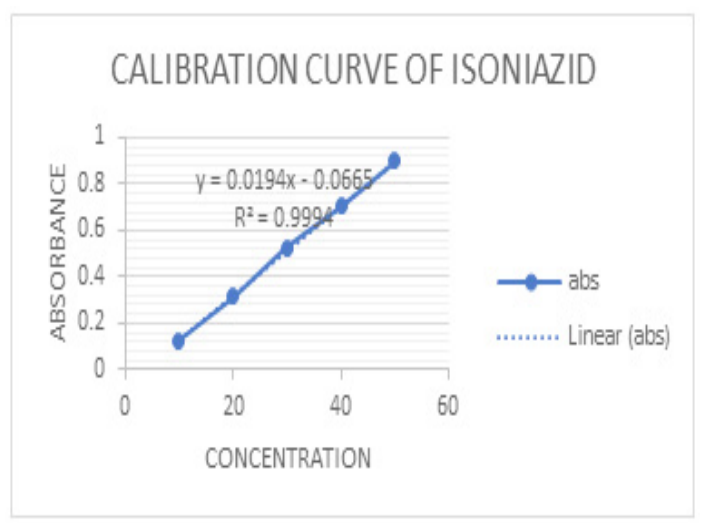

Figure 2: Calibration curve of Isoniazid.

\section{Calibration curve of Resveratrol}

Linearity for the flavonoid resveratrol was obtained at $10-50 \mu \mathrm{g} / \mathrm{ml}$ and this linearity was used for determining the concentration as represented in Figure 3.

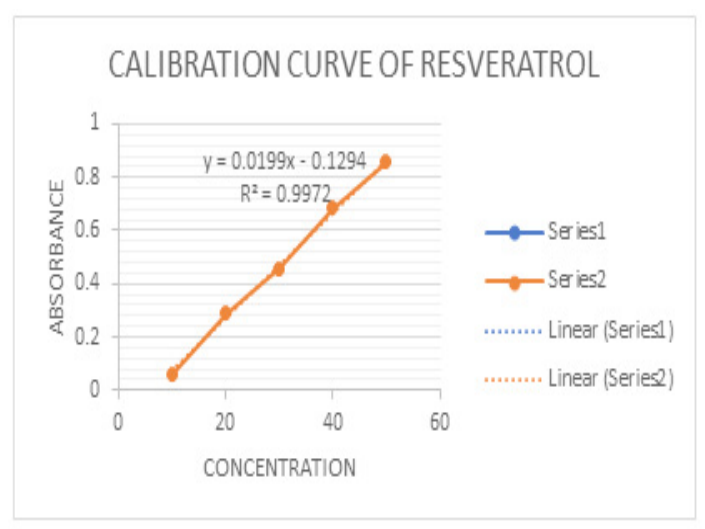

Figure 3: Calibration curve of Resveratrol.

\section{Compatibility studies}

The DSC and FTIR graphs of the samples is mentioned in Figures 4 to 10.

Differential Scanning Colorimetry:

\section{DSC of Isoniazid}

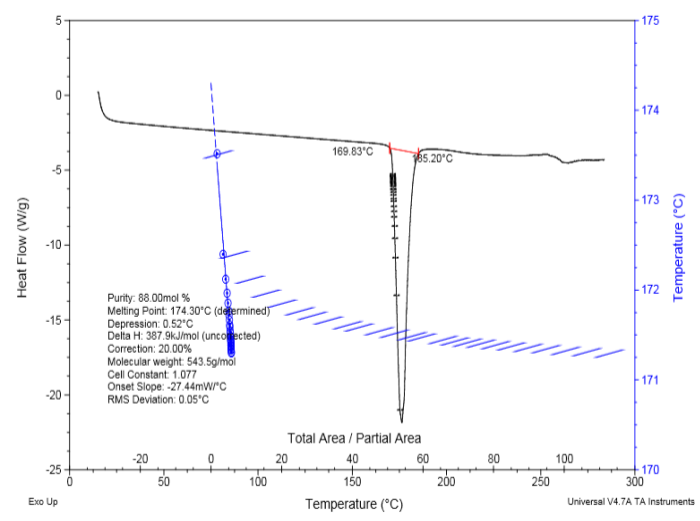

Figure 4: Representing the DSC data of Isoniazid. 
DSC of Rifampicin

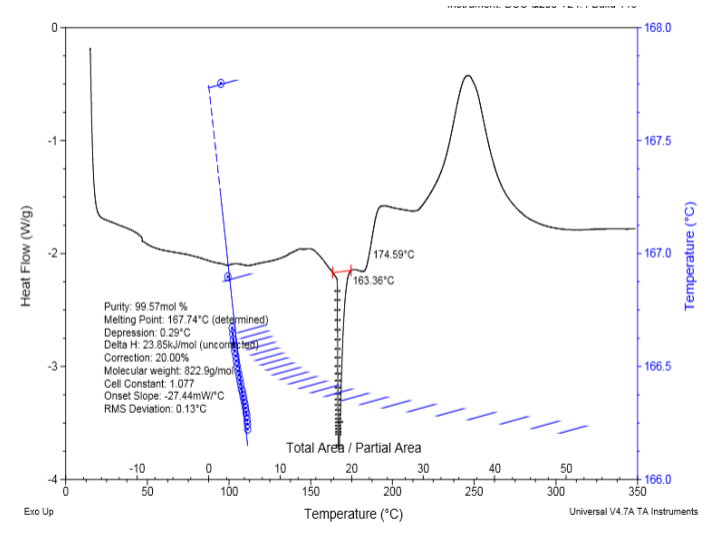

Figure 5: Representing the DSC data of Rifampicin.

\section{DSC of Resveratrol}

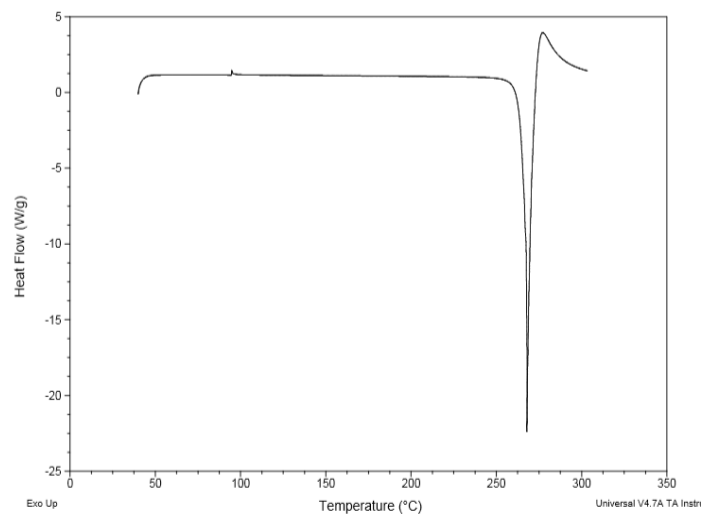

Figure 6: Representing the DSC data of Resveratrol.

\section{Fourier Transform Infrared Spectroscopy}

FTIR of Isoniazid

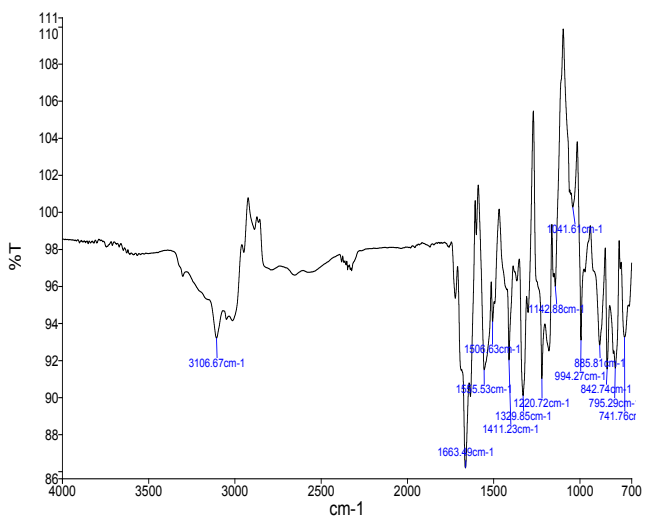

Figure 7: Representing the FT-IR data of Isoniazid.
FTIR of Rifampicin

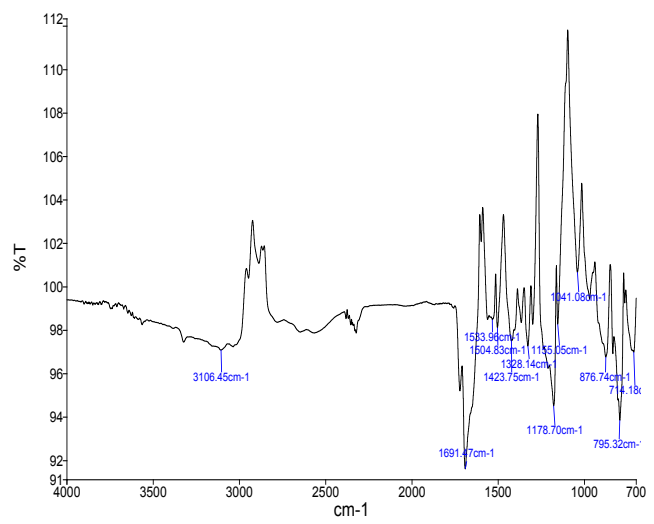

Figure 8: Representing the FTIR data of Rifampicin.

FTIR of Resveratrol

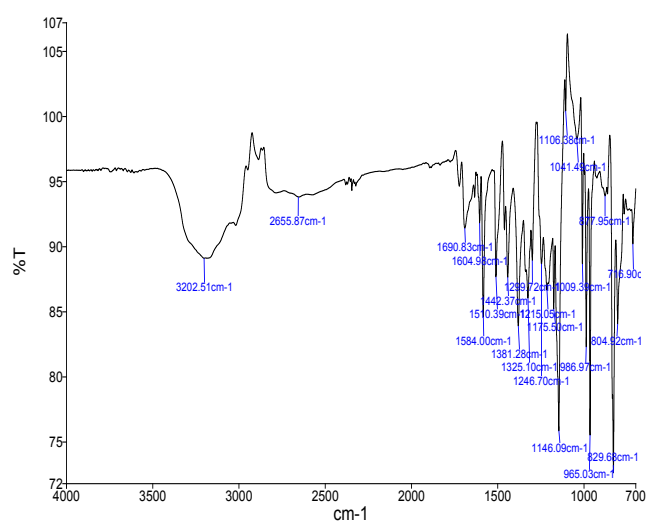

Figure 9: Representing the FTIR data of Resveratrol.

\section{FTIR of Physical Mixture}

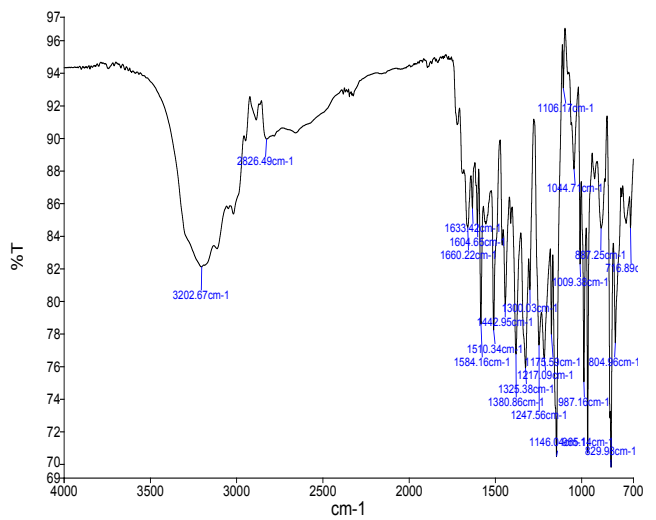

Figure 10: Representing the FTIR date of Physical mixture. 


\section{Screening of oils}

\section{Screening of rifampicin}

The graph representing the screening of rifampicin is mentioned as Figure 11.

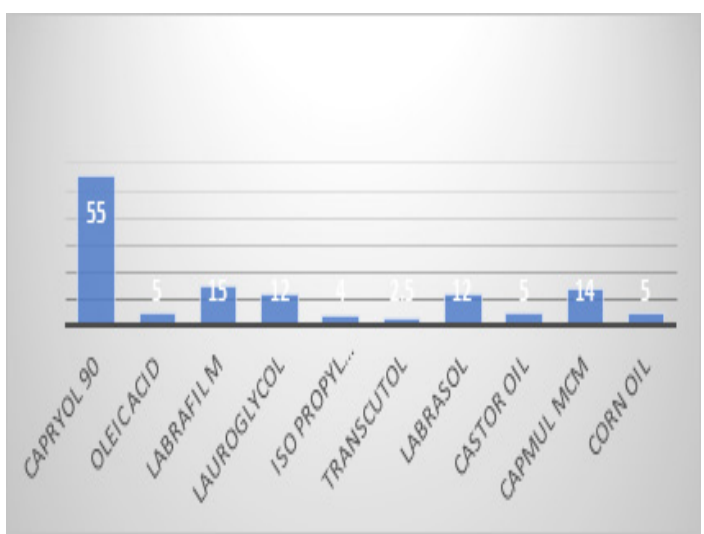

Figure 11: Representing the graphical data of solubility of rifampicin.

\section{Screening of resveratrol}

The graph representing the screening of resveratrol is mentioned as Figure 12.

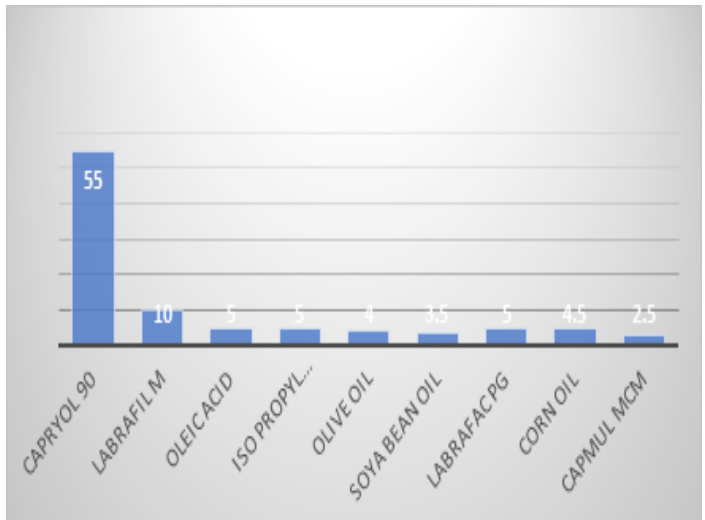

Figure 12: Representing the graphical data of solubility of resveratrol.

\section{Pseudoternary Phase Diagram of selected formulation}

The pseudoternary phase diagram of the optimised formulation A7 is shown in the Figure 13.

\section{Characterization of Nanoemulsion}

Thermodynamic stability studies

The results related to the thermodynamic stability studies are mentioned in Table 1. The samples which have passed these studies were selected for further studies.

Particle size, zeta potential and polydispersity index of the optimised formulation:

The particle size, zeta potential and polydispersity index of the optimised formulation is mentioned in the Figure 14 and 15

\section{Cell-Line Studies}

Cell-line studies were carried out to find out the $\mathrm{IC}_{50}$ values of the pure drugs as well as the formulations. The cell-line studies were carried out using C-6 cell-line as represented in the Table 2 .

\section{Nasal Toxicity Studies}

Nasal toxicity studies was carried out for placebo, Isoniazid+ resveratrol nanoemulsion and Rifampicin+ resveratrol nanoemulsion. It was found that the were no

A7

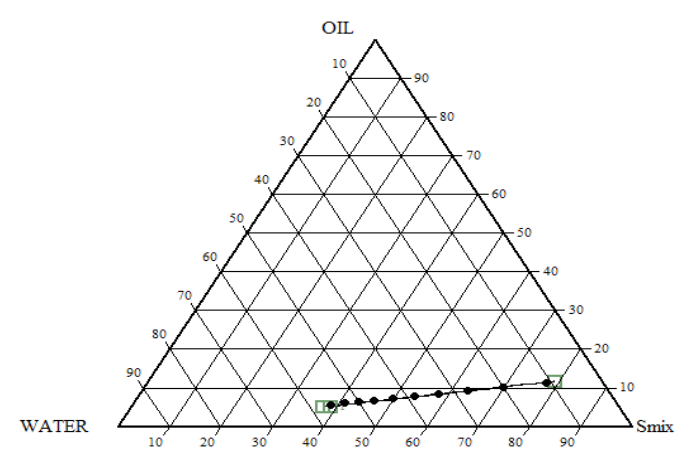

Figure 13: Pseudoternary phase diagram of A7 formulation.

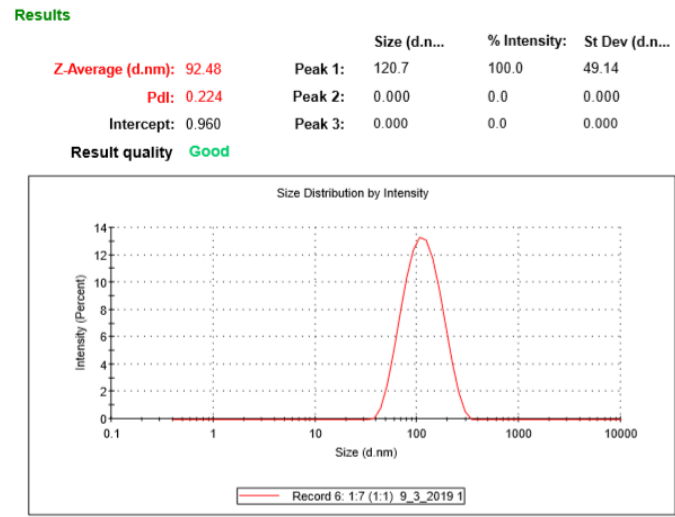

Figure 14: Representing the data of the particle size and PDI of the A7 formulation.
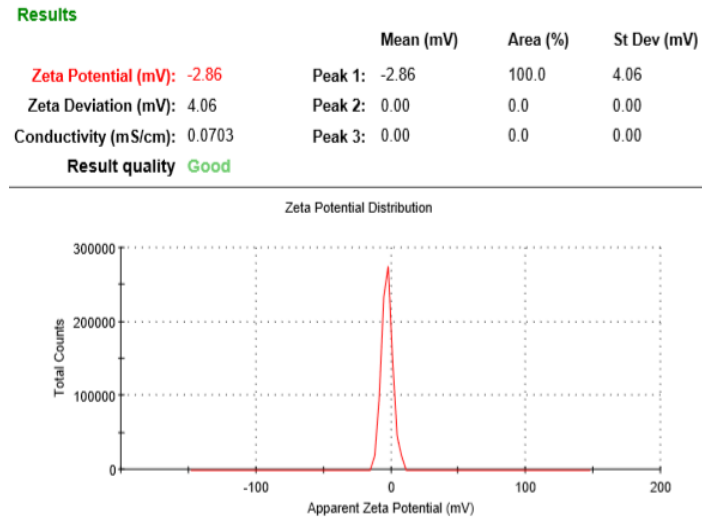

Figure 15: Representing the data of the zeta potential of the A7 formulation. 


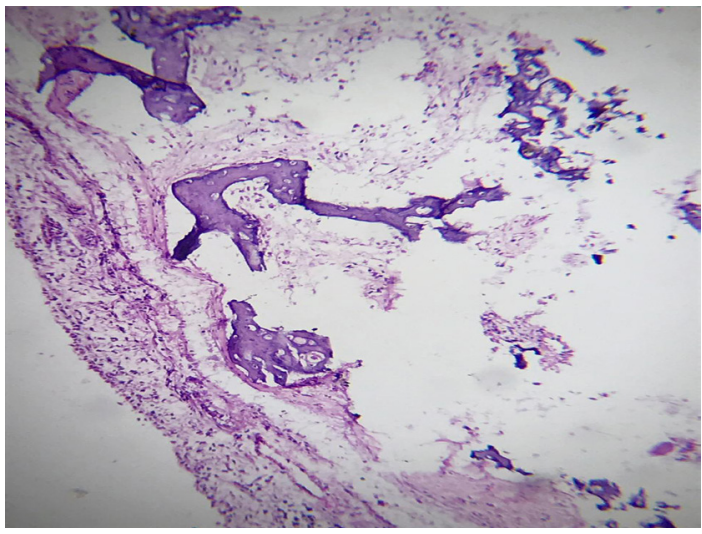

Figure 16: Nasal toxicity studies of placebo.

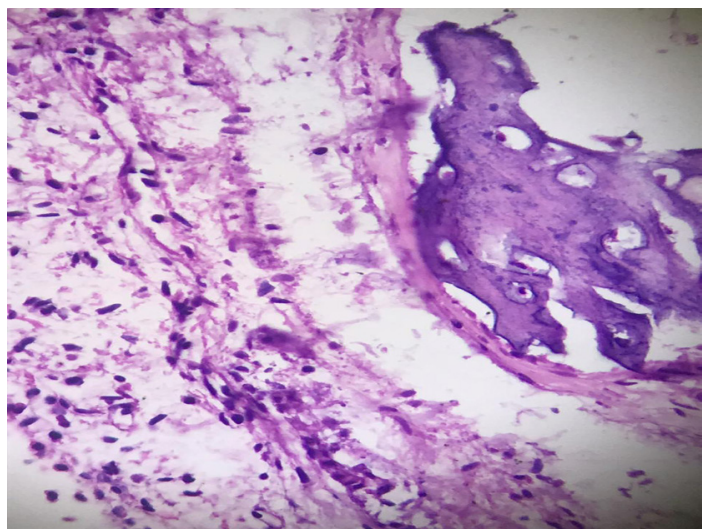

Figure 17: Nasal toxicity study for Isoniazid+ Resveratrol nanoemulsion.

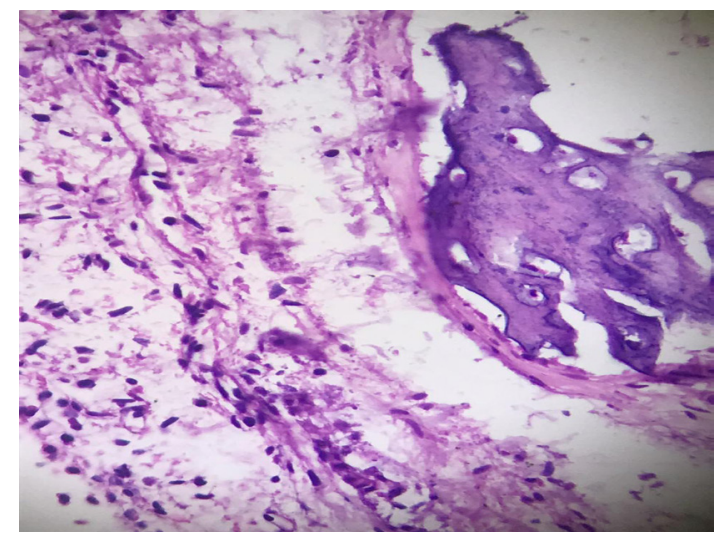

Figure 18: Nasal toxicity studies of Rifampicin + Resveratrol nanoemulsion.

indications of malignancy found in all the samples as mentioned in Figure 16,17 and 18.

\section{In vivo studies}

\section{Method Development}

\section{Standard scan of isoniazid}

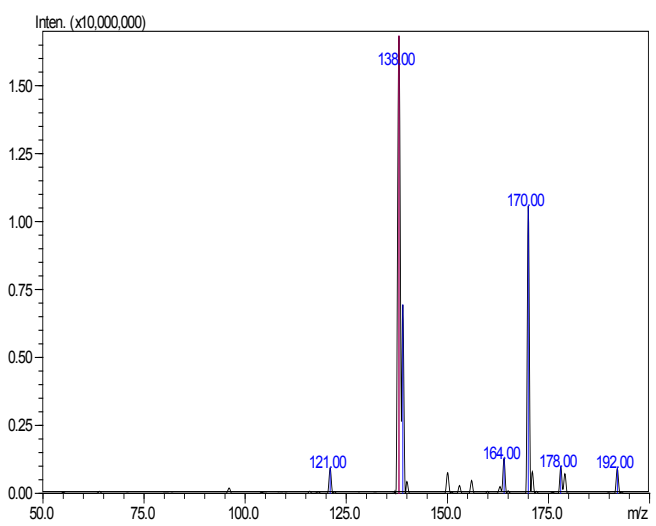

Figure 19: Graph representing the molecular weight of the isoniazid with the addition of hydrogen atom.

\section{Standard scan of rifampicin}

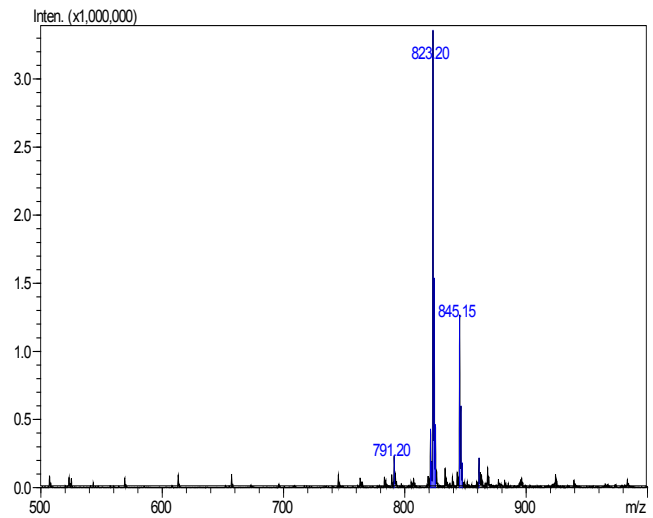

Figure 20: Graph representing the molecular weight of the rifampicin with the addition of hydrogen atom.

\section{Standard curve of resveratrol}

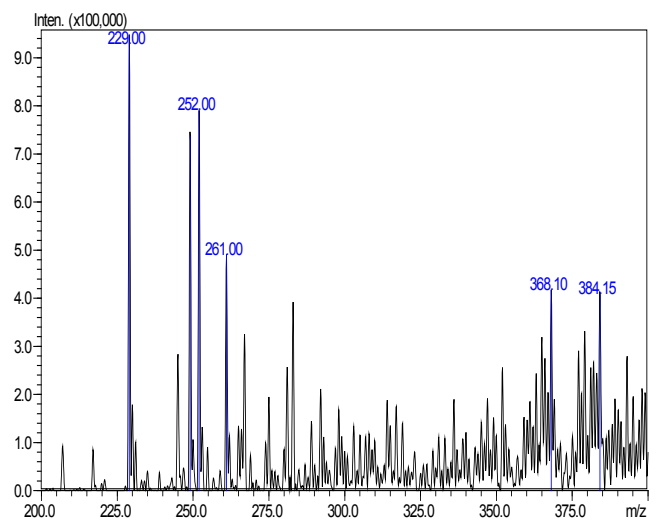

Figure 21: Graph representing the molecular weight of the resveratrol with the addition of hydrogen atom.

\section{Evaluation of the samples}

\section{Isoniazid+ Resveratrol nanoemulsion}

Around $4.5 \mathrm{mg}$ of isoniazid and $7.89 \mathrm{mg}$ of resveratrol was found in the formulation. Total amount of the drug present in the administered formulation was found to 
be $12.39 \mathrm{mg}$. Hence it was found that $82.6 \%$ of the drug has reached the brain.

\section{Rifampicin+ Resveratrol nanoemulsion \\ Isoniazid+ Resveratrol nanoemulsion}

Around $4.5 \mathrm{mg}$ of isoniazid and $7.89 \mathrm{mg}$ of resveratrol was found in the formulation. Total amount of the drug present in the administered formulation was found to be $12.39 \mathrm{mg}$. Hence it was found that $82.6 \%$ of the drug has reached the brain as mentioned in Figure 22.

\section{Rifampicin+ Resveratrol nanoemulsion}

In the formulation of nanoemulsion loaded with rifampicin and resveratrol the total amount of drug administered to the animals was $25 \mathrm{mg}$. Total amount of drug found in the brain extract was found to be $19.62 \mathrm{mg}$. Hence it was found that $78.48 \%$ of the drug has reached the brain as represented in the Figure 23.

\section{DISCUSSION}

Tuberculosis is caused by the bacteria Mycobacterium tuberculosis. The bacteria enter any part of the body. So, when the bacteria enter the brain it is termed as tuberculous meningitis. In the formulations prepared we have used the first line drugs Isoniazid and Rifampicin along with a flavonoid Resveratrol. So, we have
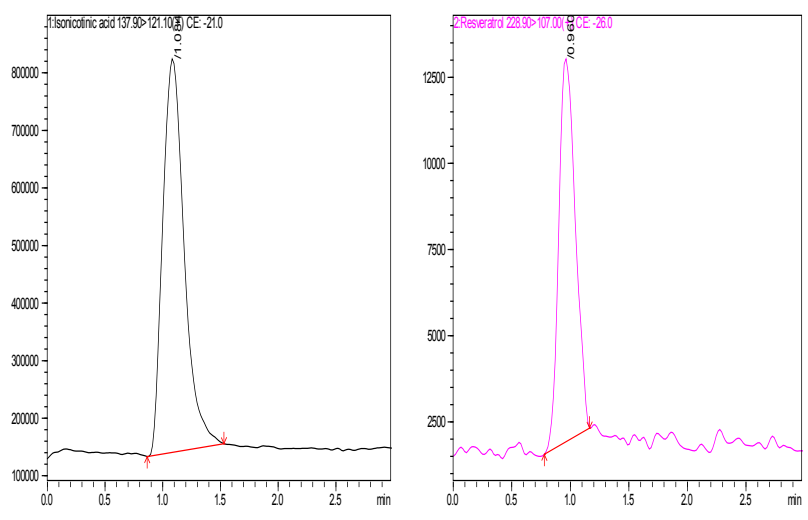

Figure 22: Representing the percentage of isoniazid and resveratrol nanoemulsion in the brain extract.
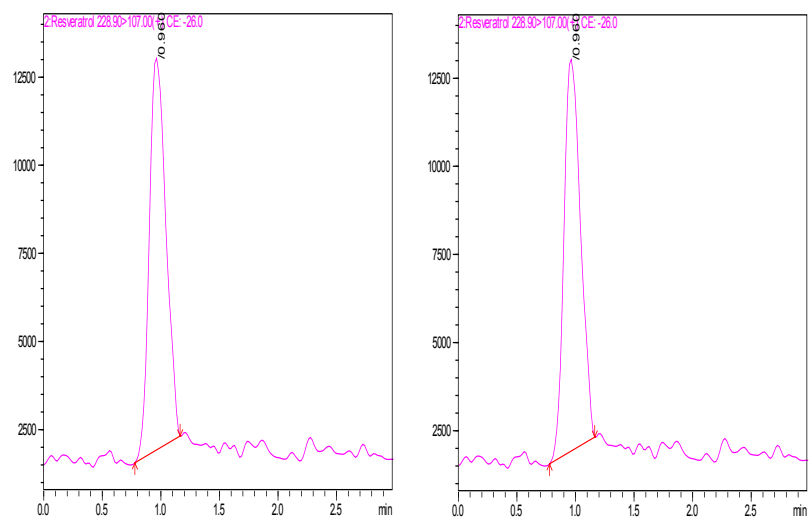

Figure 23: Graphs representing the percentage of Rifampicin+ Resveratrol nanoemulsion in the brain extract.

\begin{tabular}{|c|c|c|c|c|}
\hline \multicolumn{5}{|c}{ Table 1: Data regarding thermodynamic stability studies. } \\
\hline Formulation & $\begin{array}{c}\text { Heating and } \\
\text { cooling cycle }\end{array}$ & Centrifugation & $\begin{array}{c}\text { Freeze thaw } \\
\text { cycle }\end{array}$ & Result \\
\hline H2 & Pass & Fail & & Fail \\
\hline G8 & Pass & Fail & & Fail \\
\hline B6 & Pass & Fail & & Fail \\
\hline G7 & Pass & Fail & & Fail \\
\hline B7 & Pass & Fail & & Fail \\
\hline G5 & Pass & Pass & Fail & Fail \\
\hline D8 & Pass & Fail & & Fail \\
\hline F9 & Pass & Pass & Fail & Fail \\
\hline J6 & Pass & Pass & Fail & Fail \\
\hline G9 & Pass & Pass & Pass & Pass \\
\hline H9 & Pass & Pass & Fail & Fail \\
\hline A7 & Pass & Pass & Pass & Pass \\
\hline J8 & Pass & Pass & Pass & Pass \\
\hline J9 & Pass & Pass & Fail & Fail \\
\hline A6 & Pass & Fail & & Fail \\
\hline 13 & Pass & Pass & Fail & Fail \\
\hline H8 & Fail & Pass & Pass & Pass \\
\hline A9 & Pass & Pass & Fail & Fail \\
\hline D7 & Pass & & Fail \\
\hline
\end{tabular}


Table 2: $I_{50}$ values of all the drugs and formulations.

\begin{tabular}{|c|c|c|}
\hline SI. NO & Sample description & $\begin{array}{c}\mathbf{C} 6 \\
\text { IC }_{50} \mathbf{~} \mathbf{g} / \mathbf{m l}\end{array}$ \\
\hline 1. & Isoniazid & 62.41692 \\
\hline 2 & Rifampicin & 20.34364 \\
\hline 3 & Resveratrol & 29.63656 \\
\hline 4 & Isoniazid+ Resveratrol NE & 32.49261 \\
\hline 5 & Rifampicin+ Resveratrol NE & 13.42585 \\
\hline
\end{tabular}

conducted many pre- formulation studies. Calibration curves of all the three drugs was prepared and the graphs obtained were linear. Solubility of Rifampicin as well as Resveratrol was tested in various oils and the oil in which maximum amount of drug was soluble was selected i.e., Capryol 90. For the preparation of the formulation various surfactants and co-surfactants were tested and the surfactant and co-surfactant which was miscible with the oil was selected i.e., kolliphor and ethanol respectively. DSC of all the compounds was carried out and it was found that the compounds were pure and it formed exothermic. The DSC of the physical mixture also showed all the components in the thermogram obtained. FTIR studies were carried for all the individual compounds as well as the physical mixture. The FTIR data clearly depicts that alcohol was found in Resveratrol at 3202.51 and physical mixture at 3202.61; $\mathrm{C}=\mathrm{C}$ stretch was found in Resveratrol at 1690.83; C-H bend was found in Resveratrol at 829.68; C-H stretch in Rifampicin at $1891.47, \mathrm{C}-\mathrm{H}$ stretch of aromatic in Isoniazid at 3106.67; out of plane was in Resveratrol at 804.92 and out of plane in physical mixture at 804.96; C-C stretching in Isoniazid at 1663.49 and C-C stretching in physical mixture at 1660.22 ; N-X stretching in Isoniazid at 1142.88 and $\mathrm{N}-\mathrm{X}$ stretch in physical mixture 1140.04. Hence, it was concluded that the compounds had association and dissociation of the bonds in the physical mixture. It was also observed that the interaction between the components was not significant. Hence, it was decided to use the same for the formulation. Pseudoternary phase diagrams were constructed for different ratios of Smix from 1:1 to 1:9 and 2:1. The main hurdle faced while preparing the formulations was that the surfactant kolliphor would turn into semisolid form very frequently. So, for this reason it had to be kept in hot water bath to keep it in the liquid form. But when it was mixed with oil and co-surfactant it was stable and did not turn into semisolid. Few formulations after the addition of water in parts it formed a nanoemulsion but on further addition of water it formed an emulsion. In few formulations after addition of water it formed an emulsion but after further addition of water it turned milky. This transformation is because the surfactant and the co surfactant would get dissolved into the aqueous phase all the formulations were visually observed and the formulations which had a transparent to translucent appearance were selected. These selected formulations were further subjected to thermodynamic stability studies. Then based on the results obtained, the ratios of Smix which favoured the formulation of nanoemulsion was selected and plotted as the pseudoternary phase diagram using CHEMIX software. The ratio which formed a clear nanoemulsion was selected for the further studies. Physical characterisation of the formulations were carried out such as thermodynamic stability studies, particle size, zeta potential and poly dispersity index. The formulation which was stable and which had less particle size was selected. The inhibitory concentration $\left(\mathrm{IC}_{50}\right)$ value was influenced by the combination of drugs. This was well observed in the values obtained. The reason for this could be that the flavonoid might have played a crucial effect on the effect of Rifampicin and Isoniazid. It could be arrived that the flavonoids might give a possible synergistic effect for the drugs. Since Resveratrol being an anti-oxidant and efflux inhibitor might have sensitised the cells to respond to the drug effect. In other words, it could be explained as flavonoids are chemosensitizers. Hence the total dose in combination therapy could be reduced. Nasal toxicity studies were carried out using the mucosal membrane of the goat. The obtained membrane was divided into 3 parts and each part was put into nanoemulsion without the drugs, Isoniazid+ Resveratrol nanoemulsion and Rifampicin+ Resveratrol nanoemulsion. The reports stated that there was no malignancy found in the nasal mucous membrane. Hence it was concluded that the formulations didn't have any nasal toxicity. The prepared formulations were administered to rats intranasally and the amount of drug reaching the brain was found out using LCMS / MS method. It was found that there was increase in the amount of drug reaching the brain when the drug was administered intranasally compared to the conventional route.

\section{CONCLUSION}

Lipid based nanoemulsions were prepared as lipid substances can easily cross the blood brain barrier. Various studies were carried out and the formulation which was stable was selected. This formulation was tested for $\mathrm{IC}_{50}$ value. After obtaining the $\mathrm{IC}_{50}$ values the formulation was tested for nasal toxicity studies and the report obtained from the histopathological lab stated that there was no malignancy found. Then the formulation was administered to rats intranasally and the amount of 
drug reaching the brain was estimated using the LCMS/ MS studies and it was concluded that maximum amount of drug had entered the brain when compared to the conventional route of drug administration.

\section{ACKNOWLEDGEMENT}

The authors would like to thank the Department of Science and Technology- Fund for Improvement of Science and Technology Infrastructure in Universities and Higher Educational Institutions (DST-FIST), New Delhi for providing Nanosprey drier/ Hanson diffusion cell/ Malvern Zetasizer/ Rheometer under the DSTFIST program.

\section{CONFLICT OF INTEREST}

The authors declare no conflict of interest.

\section{ABBREVIATIONS}

BBB: Blood Brain Barrier; CSF: Cerebrospinal fluid; NE: Nanoemulsion; DSC: Differential scanning colorimetry; FTIR: Fourier Transform Infrared Spectroscopy.

\section{REFERENCES}

1. Mohammad N, KausarNeyaz M, Shilpi D. Nanotechnology-Based Approach in Tuberculosis Treatment. Tuberc Res Treat. 2017;5(12):1-12.
2. Ravindra KG, Amita J, Hardeep SM, Avinash A, Rajiv G. Drug-resistant tuberculous meningitis. Expert Rev Anti Infe. 2013;11(6):605-21.

3. Maunank S, Caitlin R. Complications of tuberculosis. Curr Opin Infect Dis. 2014;27(5): 403-10.

4. Christine J, Elizabeth W, Alasdair B, Beate K. Immunology and pathogenesis of childhood Tb. Paediatr Respir Rev. 2010;12(1):3-8.

5. Bryan RR, Michael O, Cristina AB, Thomas WM, Phillip KP. Central Nervous System Tuberculosis: Pathogenesis and Clinical Aspects. Clin Microbiol Rev. 2008;21(2):243-61.

6. Murthy JMK. Tuberculous meningitis: The challenges. Neurol India. 2010;58(5):716-22.

7. Grace EM, Edward DC. Tuberculous Meningitis: Diagnosis and Treatment Overview. Tuberc Res Treat. 2011;9.

8. Anderson NE, Somaratne J, Mason DF, Holland D, Thomas MG. Neurological and systemic complications of tuberculous meningitis and its treatment at Auckland City Hospital, New Zealand. J Clin Neurosci. 2010;17(9):1114-8.

9. Torok ME. Tuberculous meningitis: Advances in diagnosis and treatment. BMJ. 2015;113(1):117-31.

10. Vynnycky E, Fine PE. Lifetime risks, incubation period and serial interval of tuberculosis. Am J Epidemiol. 2000;152(3):247-63.

11. Digby FW, Anastasia K, Valarie M. Diversity and disease pathogenesis in Mycobacterium tuberculosis. Trends Microbiol. 2014;23(1):14-21.

12. Masahiko K, Ashish W, Hisahiro K, Muneaki H, Hiroki Y, Akinori H. Activation of Cellular Immunity in Herpes Simplex Virus Type 1- Infected Mice by the Oral Administration of Aqueous Extract of Moringa oleifera Lam. Leaves. Phytother Res. 2016;30(5):797-804.

13. Hitendra SM, Milind SM, Pankaj PN, Anshuman A. Nanoemulsion-based intranasal drug delivery system of saquinavir mesylate for brain targeting. Drug Deliv. 2014;21(2):148-54.

14. Bhanushali RS, Gatne MM, Gaikwad RV, Bajaj AN, Morde MA. Nanoemulsion based Intranasal Delivery of Antimigraine Drugs for Nose to Brain Targeting. Indian J Pharm Sci. 2009;71(6)707-9.

\section{SUMMARY}

- Rifampicin + resveratrol nanoemulsion and isoniazid + resveratrol nanoemulsion were optimised primarily by performing solubility studies. The solubility of rifampicin and resveratrol was performed in different oils and capryol 90 was selected as maximum amount of drug was soluble in this oil. Various combinations of surfactants and cosurfactants were tried but the Smix which was miscible with the oil was kolliphor and ethanol which are surfactant and co-surfactant respectively. DSC studies of the pure drugs was conducted to know the purity of the drug and the physical mixture was performed to know the compatibility. The FTIR studies of the pure drugs was conducted to know the interferences occurring in the functional groups and that of the physical mixture was carried out to know the compatibility as well as to know about the association and dissociation of the bonds.

- After the compatibility studies pseudoternary phase diagrams were constructed by taking the ratios of Smix from $1: 1$ to $1: 9$ and $2: 1$ and the ratio of the oil was kept constant. Then, thermodynamic stability studies i.e., heating and cooling cycle, centrifugation and freeze thaw cycle were performed and the formulations which were able to withstand the external stress and were stable were selected for the characterization of the formulations. The formulations which could withstand the stress were J8, G9, A9 and A7. These formulations were tested for particle size, poly dispersity index and zeta potential. The particle size of the formulations was found to be 131.1 , 96.50, 103.9,92.48 of J8, G9, A9 and A7 respectively. The polydispersity index of the formulations was found to be $0.351,0.163,0.187$ and 0.224 of J8, G9, A7 and A9 respectively. The zeta potential of the formulations was found to be $-2.90,-2.86,-2.60$ and -2.86 respectively. From these values $A 7$ was selected as the optimised formulation as it had lesser particle size when compared to the other formulations.

- Then the pure drugs isoniazid and rifampicin, flavonoid resveratrol, isoniazid + resveratrol nanoemulsion and Rifampicin + resveratrol nanoemulsion were tested for invitro toxicity studies. For these studies C-6 cell-line of the rat glioma was selected. Hence, the IC-50 values were found out. Then the formulations were subjected for nasal toxicity studies. Then these samples were sent for histopathological studies to find out the toxicity caused by the formulations and it was found that the formulations caused slight inflammation. Then in vivo studies were carried out on female wistar rats. The rats were divided into two groups. One group received Isoniazid + Resveratrol nanoemulsion and the other group received Rifampicin + Resveratrol nanoemulsion. After 45 min of administering the formulations to the animals intranasally the animals were euthanised. Then the brains of the rats were separated and the homogenised. Then the extract was centrifuged and the supernatant was collected. This supernatant was subjected for LC-MS/MS studies to know the amount of drug entering the targeted area the brain. 


\section{PICTORIAL ABSTRACT}

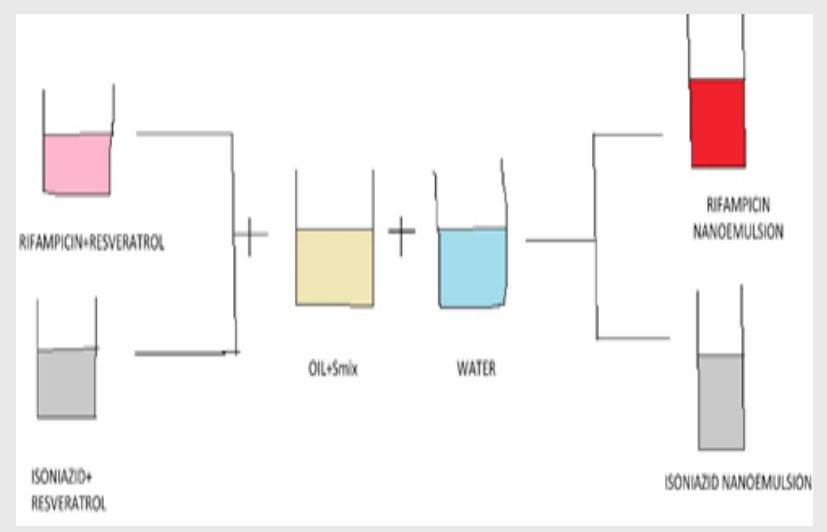

About Authors

Dr. R. Sureshkumar, is an assistant professor and Course Coordinator department of pharmaceutics. His research focuses on self nanoemulsifying drug delivery system for poorly soluble drugs for the past one decade including nanoemulgel for topical application. To his credit he has 03 patents filed under Intellectual property of India for oral delivery of statin derivatives and topical delivery of antifungal agent Terbinafine $\mathrm{Hcl}$. Published research articles in reputed journals viz., Current drug delivery, Journal of Pharmaceutical Investigation, Journal of Bioequivalence and Bioavailability, Asian Journal Pharmaceutics to name some. He is interested in developing delivery system for the plant based flavonoids which helps in the recrudescence of malaria and cancer. He is currently involved in lipid based delivery system to combat malaria and its resistance.

Cite this article: Upadhyayula SSN, Sureshkumar R, Janani SK, Karthika C. Lipid-Based Nano Formulation Approach to Target Brain for the Management of Tuberculosis through Intranasal Delivery: Formulation Development and Evaluation. Indian J of Pharmaceutical Education and Research. 2020;54(2s):s189-s199. 